\title{
Phenolics Dynamics and Infrared Fingerprints during the Storage of Pumpkin Seed Oil and Thereof Oleogel
}

\author{
Andreea Pușcaș ${ }^{1}{ }^{\circledR}$, Andruța Mureșan ${ }^{1, *}$, Floricuța Ranga ${ }^{2}{ }^{\circledR}$, Florinela Fetea ${ }^{2}$, Sevastița Muste ${ }^{1}$, \\ Carmen Socaciu ${ }^{2}$ and Vlad Mureșan ${ }^{1, * \mathbb{D}}$ \\ 1 Department of Food Engineering, Faculty of Food Science and Technology, University of Agricultural \\ Sciences and Veterinary Medicine Cluj-Napoca, 400372 Cluj-Napoca, Romania; \\ andreea.puscas@usamvcluj.ro (A.P.); sevastita.muste@usamvcluj.ro (S.M.) \\ 2 Department of Food Science, Faculty of Food Science and Technology, University of Agricultural Sciences \\ and Veterinary Medicine Cluj-Napoca, 400372 Cluj-Napoca, Romania; floricutza_ro@yahoo.com (F.R.); \\ florinelafetea@yahoo.com (F.F.); carmen.socaciu@usamvcluj.ro (C.S.) \\ * Correspondence: andruta.muresan@usamvcluj.ro (A.M.); vlad.muresan@usamvcluj.ro (V.M.); \\ Tel.: +40-264-596-384
}

Received: 4 October 2020; Accepted: 3 November 2020; Published: 5 November 2020

\begin{abstract}
Cold-pressed pumpkin seed oil is a valuable source of bioactive molecules, including phenolic compounds. Oleogels are designed for trans and saturated fats substitution in foods, but also demonstrate protection and delivery of bioactive compounds. Consequently, the present work aimed to assess individual phenolic compounds dynamics and infrared fingerprints during the ambient storage of pumpkin seed oil and thereof oleogel. For oleogels production, a $5 \%$ ternary mixture of waxes, composed by $3 \%$ beewax, $1 \%$ sunflower wax and $1 \%$ rice bran wax, was used. Phenolic compounds were extracted by traditional liquid-liquid extraction, followed by HPLC-MS quantification. FTIR (400-4000 $\mathrm{cm}^{-1}$ ) was used for characterizing and monitoring the oxidative stability of all samples and for the evaluation of intermolecular forces between oleogelator mixtures and oil. Specific wavenumbers indicated oxidative processes in stored sample sets; storage time and sample clustering patterns were revealed by chemometrics. Isolariciresinol, vanillin, caffeic and syringic acids were quantified. The main changes were determined for isolariciresinol, which decreased in liquid pumpkin seed oil samples from 0.77 (T1) to $0.13 \mathrm{mg} / 100 \mathrm{~g}$ (T4), while for oleogel samples it decreased from 0.64 (T1) to $0.12 \mathrm{mg} / 100 \mathrm{~g}$ (T4). However, during the storage at room temperature, it was concluded that oleogelation technique might show potential protection of specific phenolic compounds such as syringic acid and vanillin after 8 months of storage. For isolariciresinol, higher amounts are registered in the oleogel $(0.411 \mathrm{mg} / 100 \mathrm{~g}$ oil) than in the oil $(0.37 \mathrm{mg} / 100 \mathrm{~g}$ oil) after 5 months of ambient temperature storage (T3). Oxidation processes occurred after 5 months storage for both oil and oleogel samples.
\end{abstract}

Keywords: pumpkin seed oil; oleogels; polyphenols; HPLC-MS; Fourier transform infrared spectroscopy; chemometrics; storage follow-up

\section{Introduction}

Cold-pressed pumpkin seed oil is a dichromatic, viscous oil, abundant in valuable compounds such as unsaturated fatty acids (mostly linoleic and oleic), tocopherols, sterols, $\beta$-carotene, and lutein, the content of the bioactive compounds differing among the species and varieties, or due to climate and cultivation conditions [1,2]. The oil preserves its nutritional profile because, in general, the temperature during pressing does not exceed $50{ }^{\circ} \mathrm{C}$. However, other studies also revealed considerable contents of polyphenols in pumpkin seed oil obtained under different extraction temperatures or 
solvents, imparting overall antioxidant activity to this oil [3-5]. The numerous nutritive-pharmacological properties such as cancer prevention, anti-inflammation, anti-diabetic and lowering of cholesterol levels, are the main reasons for promoting the increase in the consumption of pumpkin seed oil $[6,7]$. Few previous studies regarding the oxidative stability of pumpkin seed oil have been conducted, especially for the cold-pressed type [4]. At this moment, it is mostly consumed as a salad dressing or encapsulated, despite its physicochemical properties which make it suitable for industrial applications too [2].

Oleogelation, a structuring technique which transforms vegetable oils into solid-like materials with the use of oleogelators, will soon be adopted as a current practice by processors and the food industry, increasing their consumption possibilities. This would be mainly due to consumers' awareness regarding healthier food and recent legislation regarding trans and saturated fat-containing products, which will be replaced by oleogels containing healthy mono or polyunsaturated oils. There are numerous possibilities of developing an oleogel, but the direct method, implying the dispersion of the structuring agents in the heated oil, followed by cooling and crystallization, is usually chosen because it is a method which is easy to up-scale. The indirect method based on an emulsion template is currently being intensively exploited and improved by researchers, but implies supplementary equipment such as spray or freeze-driers $[8,9]$. Oleogels can be formed from a wide range of structuring agents which can be classified as non-triacylglycerol gelators, more specifically crystalline particles or self-assembling structures, self-assembled fibrillar networks, polymers and inorganic compounds, or lipid-based gelators such as waxes, ceramides, phytosterols, fatty acids, fatty alcohols, and monoglycerides, etc. [10]. Recently, novel structuring agents were synthesized by enzyme catalysis [11-13]. Among them, natural waxes are cost-effective food-grade substances that demonstrated structuring capacity even at a low concentration and improved functionality when they were forming mixtures $[14,15]$. Waxes were also used to structure fish oil, in the study of oxidation of fish oil oleogel in comparison to bulk oil [16].

The versatility of oleogel process design permits the inclusion of certain fatty acids in foods, but also different liposoluble molecules and bioactive compounds, originating from the oil, from the structuring agents or added purposefully, to increase the nutritional value of the products [17-19]. $\beta$-carotene or curcumin have been included in the composition of different oleogels to exploit their health-promoting implications and to assess if model hydrophobic compounds can be protected against processing conditions and storage through oleogelation [20-23]. The usage of solely lipophilic structuring molecules can result in a negative digestibility response towards the system, and implicitly lowers the bioavailability [24]. $\beta$-sitosterol and $\delta$-oryzanol organogelators used as a mixture are an example of structuring agents which facilitate the reduction in the controversial fats through oleogelation and also provide prophylactic effects on heart diseases through cholesterol levels management, which they are able to control as phytosterols $[25,26]$. In addition, ceramides are a class of oleogelators demonstrating tumor suppressing capacity [27]. The delivery of hydrophilic bioactive compounds through oleogel was also possible, by designing functional emulsion gels containing condensed tannins, capsaicin or inulin [28-30]. Polyphenols are able to form stable crystals which stabilize emulsion template oleogels and transform the oleogel into a functional matrix due to their biological activity [8,31].

Polyunsaturated vegetable oils, such as pumpkin seed oil, which are already abundant in polyphenols or other biologically active compounds, are more desirable for oleogel formulation and inclusion in food products such as meats, diary, pastry, and confectionary, to improve their nutritional profile. Moreover, oil blends should be developed, since the addition of only $1 \%$ cold-pressed pumpkin seed oil to sunflower oil demonstrated an antioxidant effect [32].

Most of the research focuses on evaluating if the oleogel can mimic the rheological, textural, and sensory properties of the fat and their inclusion in foods [33,34]. The present study follows the impact of oleogelification on the biologically active compounds delivered by the oil, namely the specific polyphenols, which might be altered by the oleogelation processes such as mixing, heating, or during UV storage $[33,34]$. Regarding the chemical and nutritional characterization of different pumpkin 
seed oils, it has been reported repeatedly that the fatty acid composition of the oil consists of monoand poly-unsaturated fats, the double bonds inside the alkyl chains of the fatty acids making the pumpkin seed oil prone to oxidation [35,36]. Additionally, a decrease in the total polyphenols of the pumpkin seed oil stored up to 120 days was reported [37]. Consequently, the present work aimed to assess individual phenolic compounds dynamics in fresh state (T1) and during the ambient storage of pumpkin seed oil and thereof oleogel, after one month (T2), five months (T3) and eight months (T4), but also the infrared fingerprints and chemometrics, in order to study oxidation and the implication of the oloegel in retarding the phenomenon or the polyphenols' antioxidant effect.

\section{Materials and Methods}

\subsection{Materials}

Cold-pressed pumpkin seed oil was purchased from Luna Solai, a Romanian producer. The waxes are natural and were kindly provided by Kahlwax (Trittau, Germany). HPLC filters, Chromafil, with a pore size of $0.45 \mu \mathrm{m}$ and filter diameter of $13 \mathrm{~mm}$, were also used for extractions. Hexane and $\mathrm{MeOH}$ were purchased from Merck (Darmstadt, Germany). Acetonitrile and acetic acid used for HPLC-MS analysis were purchased from Merck (Darmstadt, Germany). All other chemicals were of analytical grade.

\subsection{Oleogel Production}

For oleogel production, a 5\% wax ternary mixture was used to gel cold-pressed pumpkin seed oil. As a structuring agent, a ternary mixture of waxes was chosen since it was previously found that it leads to the formation of oleogels with excellent physical properties. The mixture was formed by $3 \%$ beeswax, $1 \%$ sunflower wax and $1 \%$ rice bran wax. The wax and the oil were heated above the highest melting point of the waxes $\left(80^{\circ} \mathrm{C}\right)$ on a magnetic hot stirrer plate. The melted mixture was poured in aliquots of $30 \mathrm{~g}$ (OD), and these were closed and stored at room temperature under light exposure along with the liquid oil (UD), with different transparent polypropylene vessels (50 $\mathrm{mL}$ volume) being used for sampling each storage time (fresh-T1, one month- T2, five months- T3 and eight months-T4).

\subsection{Phenolic Compounds Extraction}

Phenolic compounds extraction consisted of a traditional liquid-liquid extraction as mentioned Ricciutelli et al. [38]. A volume of $3 \mathrm{~mL}$ of the oil or oleogel sample and $3 \mathrm{~mL}$ hexane were transferred into a $15 \mathrm{~mL}$ centrifuge tube which was vortexed (MX-S, Dlab Scientific Co., Beijing, China) for $30 \mathrm{~s}$, until the mixture was homogenized. A volume of $5 \mathrm{~mL}$ of methanolic solution (3:2) was added, then it was subjected to sonication (Bandelin SONOREX, Sigma Aldrich, Berlin, Germany) for 15 min and centrifuged (DM0412 Dlab Scientific Co., Beijing, China) for $10 \mathrm{~min}$ with $3000 \mathrm{rot} / \mathrm{min}$. The supernatant was disposed and $3 \mathrm{~mL}$ hexane was re added to the methanolic pellet in order to dilute the remaining lipidic compounds and the vortex, sonication and centrifugation steps were repeated for 2-3 times, until the supernatant was clear. Furthermore, a concentration step at $35^{\circ} \mathrm{C}$ under reduced pressure was conducted (Rotavapor Heidolph). The evaporated samples were solubilized in $1 \mathrm{~mL}$ of methanol, filtered through $0.45 \mu \mathrm{m}$ Millipore nylon filter and used for HPLC-MS analysis.

\subsection{HPLC-MS Quantification of Specific Phenolic Compounds}

High-performance liquid chromatography (HPLC) remains one of the most important analytical tools for fingerprinting and quantifying bioactive compounds. HPLC analysis was performed on Agilent 1200 system equipped with a quaternary pump, a degasser DGU-20 A3 (Prominence), an autosampler, an UV-VIS detector with photodiode (DAD), coupled with a single-quadrupole Mass Detector Agilent model 6110 (Agilent Technologies, Santa Clara, CA, USA). The compound separation was conducted on Eclipse XDB C18 column $(5 \mu \mathrm{m}, 4.6 \times 150 \mathrm{~mm})$. The mobile phase consisted of two solvents, A and 
$\mathrm{B}$, injected at $25^{\circ} \mathrm{C}$ for $30 \mathrm{~min}$, at a flow rate of $0.5 \mathrm{~mL} / \mathrm{min}$ and a gradient which is described in the following, together with the solvents. The mobile phase composition: solvent A-bi-distilled water and $0.1 \%$ acetic acid/acetonitrile (99/1) $v / v$, B - acetonitrile and acetic acid $0.1 \%$. The solvent gradient applied was as follows: $0 \mathrm{~min}, 5 \% \mathrm{~B} ; 0-2 \mathrm{~min}, 5 \% \mathrm{~B} ; 2-18 \mathrm{~min}, 5-40 \% \mathrm{~B} ; 18-20 \mathrm{~min}, 40-90 \%$ B; 20-24 min, 90\% B; 24-25 min, 90-5\% B; 25-30 min, 5\% B. The chromatograms were monitored at 280 and $340 \mathrm{~nm}$, respectively. The mass spectrometric data were obtained using a single-quadrupole 6110 mass spectrometer (Agilent Technologies, Chelmsford, MA, USA) equipped with an ESI probe. The measurements were performed in the positive mode with an ion-spray capillary voltage of $3000 \mathrm{~V}$, and a temperature of $300{ }^{\circ} \mathrm{C}$. The nitrogen flow rate was $7 \mathrm{~L} / \mathrm{min}$. Data were collected in full scan mode within the range 100 to $1200 \mathrm{~m} / \mathrm{z}$. The data reading and acquiring was done using Agilent ChemStation software.

\subsection{Fourier Transform Infrared Spectroscopy}

The FTIR spectra were acquired with Shimadzu IR Prestige-21 equipment (Shimadzu Corporation, Kyoto, Japan) in order to evaluate the oxidative stability of oleogel and reference oil, but also to analyze the intermolecular forces between the wax ternary mixture and the oil. The oil and the oleogel samples were placed directly on the sampling device equipped with a single reflection attenuated total reflectance (ATR). Spectra were scanned in the $600-4000 \mathrm{~cm}^{-1}$ wave number range with a resolution of $4 \mathrm{~cm}^{-1}$ and 64 scans. The spectral data were processed with Origin PRO8. To remove noise and eliminate background effects, the acquired spectral values were processed with standard normal variate (SNV) and the scatter effects were removed by centering and scaling each individual spectrum.

\subsection{Chemometrics}

By principal component analysis (PCA), using the Unscrambler 10.1 Software, version 10.1 (Camo Software AS, Oslo, Norway), the variability and similarities between samples registered during FTIR analysis were depicted. The PLS analysis (partial least squares) was also performed in order to reveal the possible relations existing between the presence of the polyphenols in the sample and the resulting FTIR spectra during the 8 months of storage, both for the oils and oleogels.

\subsection{Statistical Analysis}

Two-way analysis of variance (ANOVA) using Minitab Statistical Software v.19 (Minitab Inc., State College, PA, USA) was performed in order to assess the influence of pumpkin seed oil's physical state (oil vs. oleogel) and storage time on individual phenolic concentrations; for each compound, Tukey's comparison tests were performed at a 95\% confidence level.

\section{Results and Discussion}

\subsection{Phenolic Compounds Extraction and Individual Characterization}

The samples were subjected to a traditional liquid-liquid extraction, with hexane and methanolic solution as solvents with no further acid hydrolysis. Then, compound identification and peak assignments, or the total polyphenolic content, were assessed based on the retention times, UV-VIS spectra and also comparing with ESI-MS results. The HPLC-MS analysis allowed the identification and quantification of individual polyphenolic components and revealed the presence of isolariciresinol, vanillin, caffeic and syringic acids, based on the parameters described in Table 1, both in the oil and oleogel samples.

The extraction method proved efficiency, since in another study, where the phenolic compounds extracted with the solid phase method (SPE) from a sample consisting of cold-pressed pumpkin seed oil produced for commercial purpose could not be individually identified [5]. The original method of solid phase extraction (SPE) of the phenolic compounds is not suitable for pumpkin seed oil and needs adaptation, but is preferred, because is a premise of good separation of the interest compounds, 
according to a research which investigates different extraction methods and protocols [4]. The lack of accuracy of the Folin-Ciocalteu method for the calculation of total polyphenolic content of pumpkin seed oil was also discussed and seems to be due to the influence of the carotenoids on the SPE extraction and on the method, the bleaching of oil before this measurement not being indicated because it affects specific polyphenolic compounds and would not solve the inconvenience [4].

Table 1. Phenolic compounds identification in cold-pressed pumpkin seed oil by HPLC-MS.

\begin{tabular}{cccc}
\hline Retention Time (min) & UV Max & {$[\mathbf{M}+\mathbf{H}]+\mathbf{( m / z )}$} & Identification \\
\hline 12.12 & 320 & 181 & Caffeic acid \\
15.05 & 280 & 198 & Syringic acid \\
16.31 & 265,320 & 153 & Vanillin \\
26.27 & 280 & 360 & Isolariciresinol \\
\hline
\end{tabular}

In the present study, the total phenolic contents of the cold-pressed pumpkin seed oils and their correspondent oleogels were also assessed by HPLC-MS and the results were $1.087 \mathrm{mg} / 100 \mathrm{~g}$ for the oil at fresh state and $0.999 \mathrm{mg} / 100 \mathrm{~g}$ for the oleogel (T1), a similar content being reported by the literature for the oil [39]. From our knowledge, this is the first time when the total polyphenols are analyzed for a pumpkin seed oil oleogel. The content of the individual and total polyphenols in the cold-pressed pumpkin seed oil and oleogel during storage, expressed as $\mathrm{mg} / 100 \mathrm{~g}$ oil, is represented in Figure 1. For all individual phenolics (caffeic acid, syringic acid, vanillin, isolariciresinol, as well as total phenolics), the two-way ANOVA revealed high significant differences $(p<0.05)$ for both factors (oil physical state and storage time) as well as their interaction. Consequently, the oil state-storage time interactions were analyzed by several one-way ANOVA tests and compared by Tukey's method. Thus, the influence of oleogelation and storage duration over the phenolics dynamics is depicted by Figure 1, including the statistical significance.

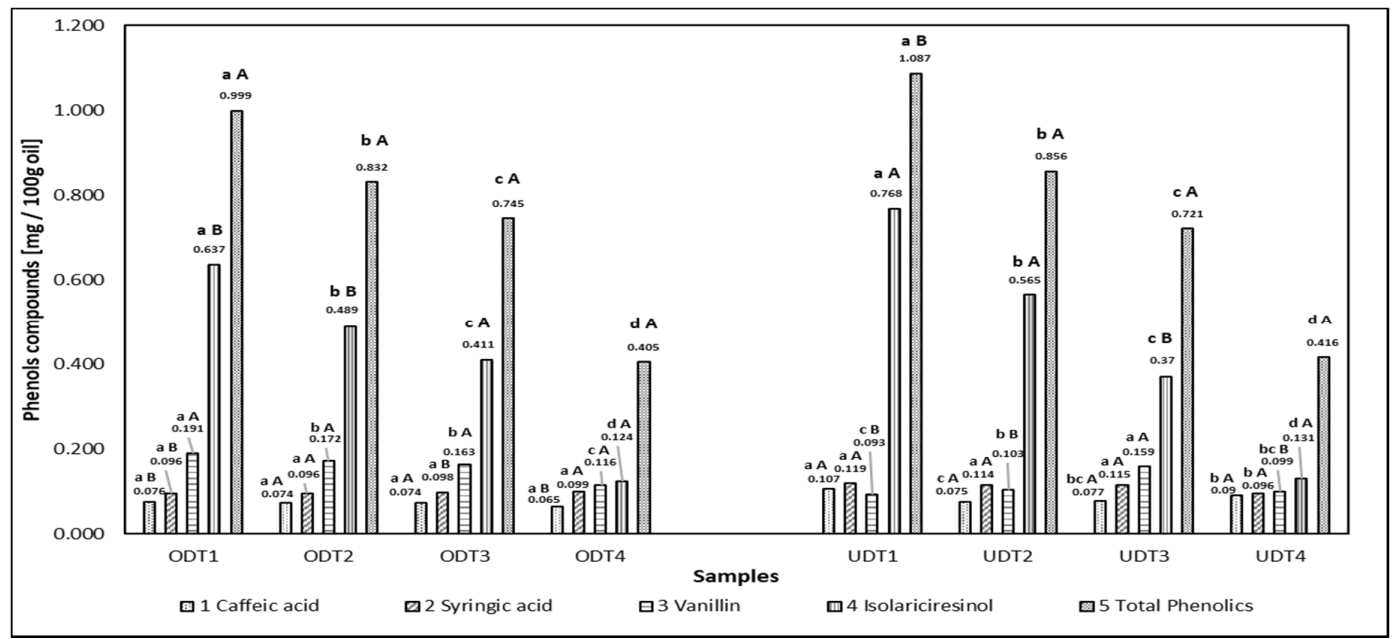

Figure 1. Individual and total phenolic compounds detected by HPLC-MS in cold-pressed pumpkin seed oil (UD) and oleogel (OD) fresh (T1) but also stored for one (T2), five (T3) and eight months (T4) at room temperature. For the same compound, lower-case letters within each sample group (oleogel or oil) indicate no significant difference $(p>0.05)$; for the same compound and storage time, upper-case letters between each sample type (oleogel vs. oil) indicate no significant difference ( $p>0.05)$.

For caffeic acid, syringic acid and isolariciresinol lower amounts were detected in the oleogel samples in comparison with the oil. Isolariciresinol is a lignan commonly identified in the pumpkin seed, but which is present in lower amounts in the oil too. In addition, it might be thermolabile, since in oleogel it is present in slightly lower amounts than in the oil at fresh state. For the cold-pressed pumpkin seed oil investigated in our research, isolariciresinol was the most abundant polyphenolic 
compound detected $(0.768 \mathrm{mg} / 100 \mathrm{~g}$ in the fresh oil). The content of another oligomer of this lignan, the secoisolariciresinol, it is reported to be lowered both in seed and oil, because of the roasting process [4]. From the class of hydroxycinnamic acids, the caffeic acid was identified $(0.107 \mathrm{mg} / 100 \mathrm{~g}$ oil in the fresh state) and from the class of hydroxybenzoic acids, the syringic acid was present in an amount of $0.119 \mathrm{mg} / 100 \mathrm{~g}$ oil. In the oleogel samples, caffeic and syringic acids showed no statistically significant differences during storage $(p>0.05)$, while in the oil samples, a varying trend was noticed, as depicted in Figure 1. An overall statistically significant higher content of vanillin $(p<0.05)$, a compound of the class of hydroxy benzaldehydes, is present in the oleogel samples, in each moment of storage, in comparison to the oil samples. In the oleogel, $0.191 \mathrm{mg} / 100 \mathrm{~g}$ was detected, while in the fresh oil it was $0.093 \mathrm{mg} / 100 \mathrm{~g}$, thus the thermal treatment might promote the release of this compound. The amount of vanillin in the oleogel samples decreased during storage and a statistically significant difference between samples was revealed; in the oil samples, the vanillin concentrations were varying during the eight months storage (Figure 1).

The content of the total polyphenols decreased during storage to less than half, both for the oil and the oleogel samples, the main changes being determined for isolariciresinol, which statistically significant decreased $(p<0.05)$ in liquid pumpkin seed oil samples from 0.77 (T1) to $0.13 \mathrm{mg} / 100 \mathrm{~g}$ (T4), while for oleogel samples from 0.64 (T1) to $0.12 \mathrm{mg} / 100 \mathrm{~g}$ (T4). Therefore, during the storage at room temperature, it can be observed that oleogelation technique might show potential protection of overall phenolic compounds, especially for the syringic acid and vanillin, higher content being registered in the oleogel sample in comparison to the oil, in samples stored at room temperature for 8 months (T4). For isolariciresinol, statistically significant higher amounts $(p<0.05)$ are registered in the oleogel $(0.411 \mathrm{mg} / 100 \mathrm{~g}$ oil) than in the oil $(0.37 \mathrm{mg} / 100 \mathrm{~g}$ oil) after 5 months of ambient temperature storage (T3).

\subsection{Fourier Transform Infrared Spectroscopy}

FTIR spectroscopy coupled with the chemometrics techniques is a recently intensively exploited method in the fast, simple and non-invasive analysis of edible oils. It is suitable for determining both the composition and the quality of oils. The FTIR spectra, scanned using attenuated total reflectance mode, displayed both for the oil and oleogel samples, raw and SNV-treated, peaks characteristic for vegetable oils. For instance, the peak at $3007 \mathrm{~cm}^{-1}$ is denoting the poly unsaturation of the oil, sharp peaks also being registered in the 2924, 2852, and $1745 \mathrm{~cm}^{-1}$ region, to reveal the abundance of triglycerides in the samples (Figure 2). At the lower frequencies, the band near $720 \mathrm{~cm}^{-1}$ is assigned to the rocking vibration of the $\mathrm{CH}_{2}$ group which build up the long-chain mono and polyunsaturated fatty acids of the pumpkin seed oil. The broad peak at $1160 \mathrm{~cm}^{-1}$ might be due to C-O stretching of a tertiary alcohol or even due to the presence of the phenolic compounds. Other studies describing the FTIR spectra of pumpkin seed oil also registered smaller intensity peaks in the region of 1695,1460 , $1378,1237,1160,1110,1097,950$ ' and $850 \mathrm{~cm}^{-1}$, some small shifts in the peaks to left or right being registered due to the particularity of the oils composition [40-42].

During the oleogel formation, the cold-pressed pumpkin seed oil was subjected to a thermal treatment of $80^{\circ} \mathrm{C}$ for up to $5 \mathrm{~min}$ and to shearing, and even if oleogelation does not affect the chemical properties of oil, different molecular arrangements occur due to the crystallization of the ternary mixture of waxes and the oil, during cooling, affecting the appearance of the spectra, depending on the oil and the oleogelation technique. Beside changes in intensities, it can be affirmed that there was no variability in the peak appearance or shifts for the oleogels in comparison to the oils (Figure 2), which indicates that physical interactions, and not changes in the chemical structure, are the reason for oleogel formation. Usually in the study of oleogels, medium intensity bands in the $3550-3450 \mathrm{~cm}^{-1}$ range indicate intermolecular bounding and $3570-3540 \mathrm{~cm}^{-1}$ range bands indicate the presence of some intramolecular hydrogen bonds in the FTIR spectra [43]. The spectra of the wax based oleogels usually lack a well-resolved band in this region [44]; also, other authors evaluate the bands at $2924 \mathrm{~cm}^{-1}$ specific for the lipid acyl-chains, which in our study for the cold-pressed pumpkin seed the oleogel sample displays lower intensity, and thus a slightly higher conformational ordering than the oil [45]. 


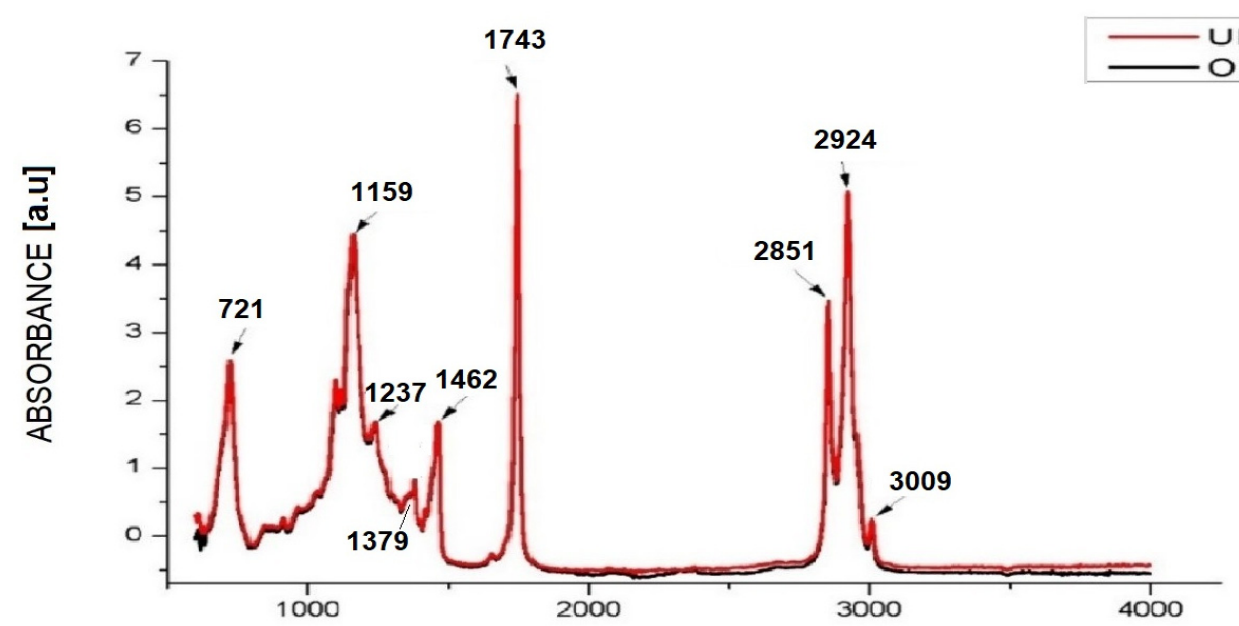

\section{WAVE NUMBER $\left[\mathrm{CM}^{-1}\right]$}

Figure 2. FTIR raw spectra of pumpkin seed oil (UDT1) and oleogel (ODT1) in fresh state (T1).

It is already concluded that oxidation processes during heating occur more slowly in oils extracted from 4-12 min microwave roasted pumpkin seeds in comparison to oils from unroasted seeds [46]. In the present study, the effect of thermal treatment on the oxidative stress of the oleogel samples is evaluated, by comparing the different intensities of several key absorbances for the oil and oleogel samples at $\mathrm{T} 1$, in fresh state (Figure 2). The heat-induced oxidation can be monitored at the $3050-2800 \mathrm{~cm}^{-1}$ or $1743-1465 \mathrm{~cm}^{-1}$ spectral region, where slight differences in the values of the intensities were registered for the oil and oleogel samples in fresh state [46]. In the $1500-1300 \mathrm{~cm}^{-1}$ region, the bending vibration of methyl and methylen groups can be analyzed. For the oleogel samples in fresh state, higher intensities were registered and this might be due to the waxes' contribution, which are derived from mixtures of long-chain hydrocarbons.

Oil oxidation is a set of natural phenomena governed by intrinsic and extrinsic factors, determining the shelf life, nutritional and sensorial properties of edible oils. This is a multidimensional result of the fatty acid composition of oil, and the presence of antioxidant components such as phenolic compounds, concurring with oxygen, light or heat [47]. The samples analysed by HPLC-MS for the quantification of total polyphenolic compounds displayed a decrease of almost $50 \%$ during the 8 months of storage; therefore, higher oxidation of the oils and oleogels is expected to be registered as the storage time increases. Both the oil and oleogel samples stored for 5 months display peaks overlaid with the peaks of the rest of the samples (at fresh state and stored for 1 months) and, in conclusion, at that moment, oxidation processes were not advanced. In the raw spectra, the intensity of the peaks displayed for the oleogel sample stored for 8 months (ODT4) are lower than the rest of the samples in the following regions: 2924, 2850, 1743, and $1157 \mathrm{~cm}^{-1}$, but did not decreased for 3008, 1465, and $715 \mathrm{~cm}^{-1}$.

The raw and SNV-treated FTIR spectra were registered for both the oil (Figure 3a,b) and oleogel samples (Figure 3c,d) in different moments of the storage period: fresh (T0), 1 month (T1), 5 months (T3) and 8 months (T4) in order to follow the oxidation during the 8 months of storage and to reveal the influence of the total polyphenols. The spectra started to reveal significant differences in terms of the intensity and position of the relevant bands (the shifts were not large but visible). For the SNV-treated spectra, the increase in a peak can be seen in regions of $1743 \mathrm{~cm}^{-1}$ for the oleogel in comparison to the oil, which is due to the formation of carbonylic compounds and cis allylic during oxidation. For the raw spectra of the oleogel, the peak at $3008 \mathrm{~cm}^{-1}$ was decreased because of the decrease in cis allylic $(\mathrm{C}=\mathrm{CH})$ bonds and is slightly shifted to the right, in comparison to the peak registered for the oil samples. 


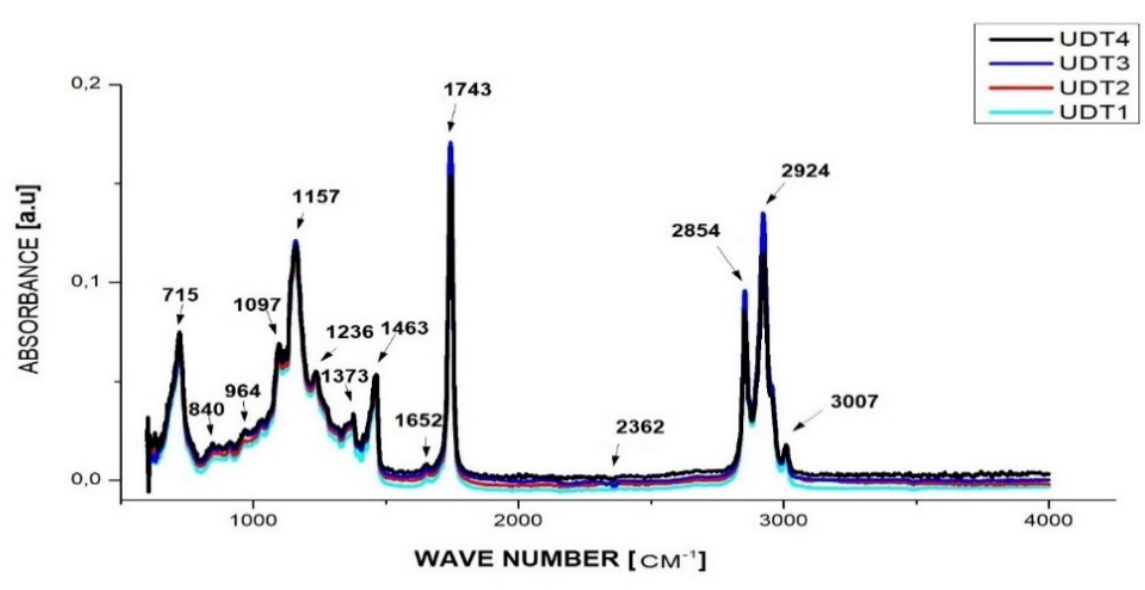

(a)

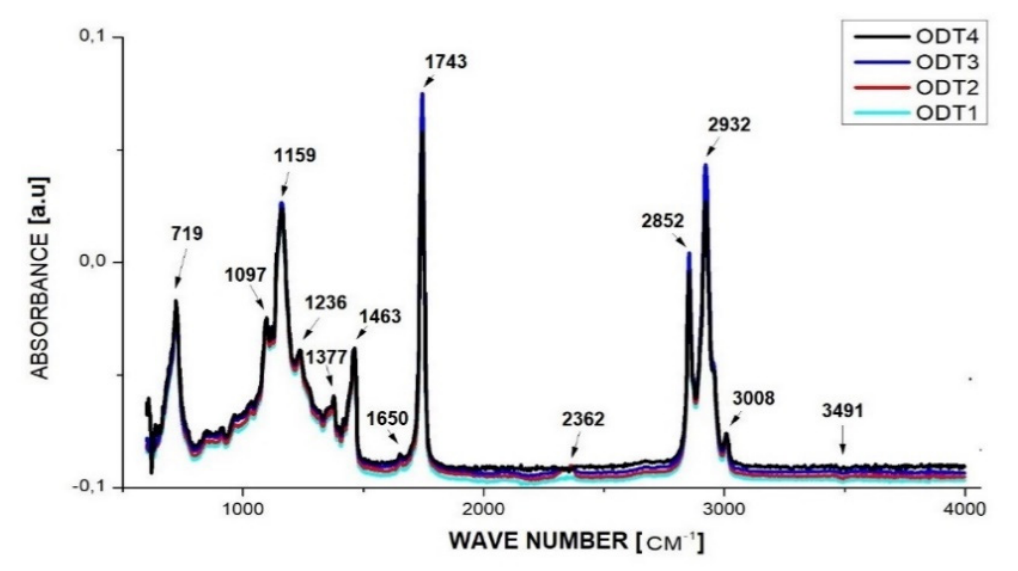

(c)

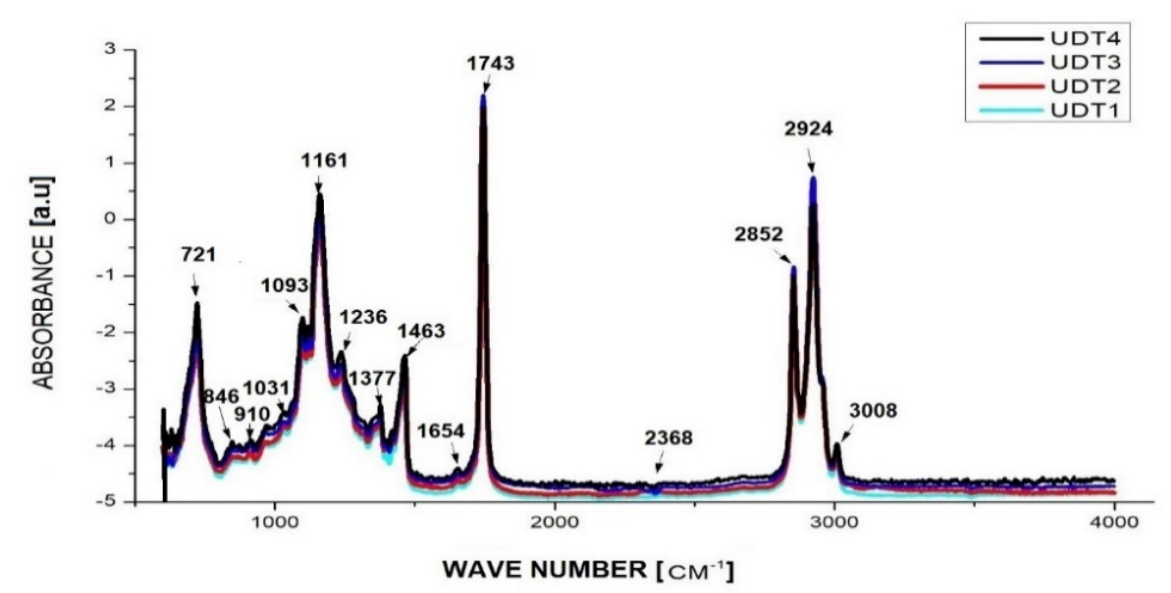

(b)

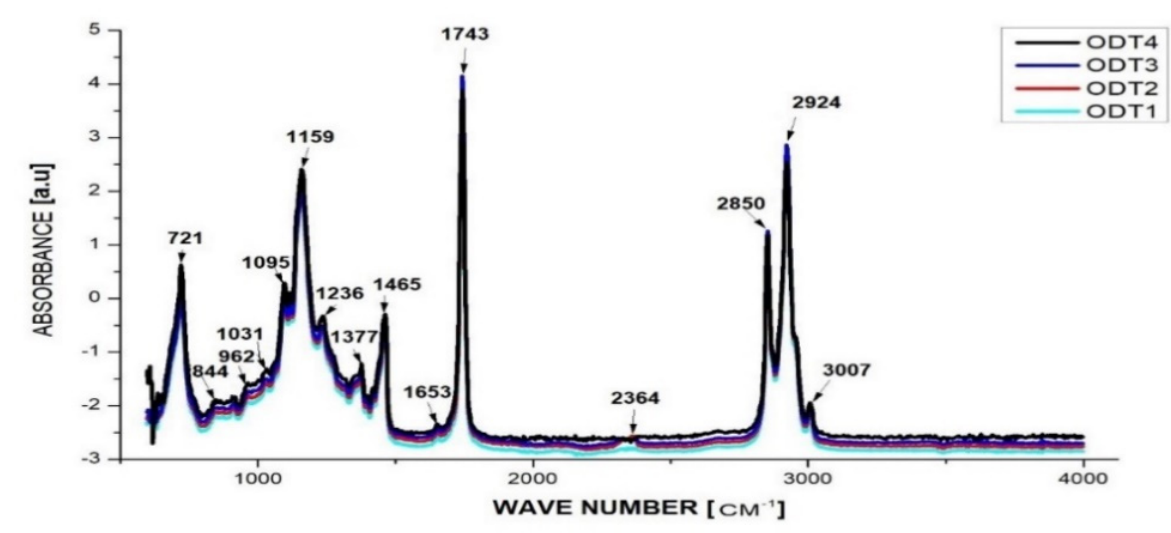

(d)

Figure 3. FTIR spectra of pumpkin seed oil (UD) and oleogel (OD) at fresh (T1), and stored one month (T1), five (T2) and eight months (T4) at room temperature: (a) oil raw spectra; (b) standard normal variate (SNV)-treated oil spectra; (c) oleogel raw spectra; (d) SNV-treated oleogel spectra. 
During oxidation, double bonds will be converted in single bonds, as a first step to hydroperoxide formation, and this can be confirmed by an increase in the ratio of intensities $2850 / 3008 \mathrm{~cm}^{-1}$. Figure 4 depicts the ratio of intensities $2850 / 3008 \mathrm{~cm}^{-1}$ during the storage of oil and oleogel samples. It was established in order to inspect the effect of the oleogelation related to storage and its potential protection against oxidation or loss of bio-active compounds, by comparing the 8 months stored oleogel and oil samples. Figure 4 indicates a more intense oxidation for the oleogel sample after 5 months of storage in comparison to the oil. The current study hypothesis that oleogelation protects the cold-pressed pumpkin seed oil from oxidation or from the loss of polyphenols is thus invalidated for samples stored longer than 5 months.

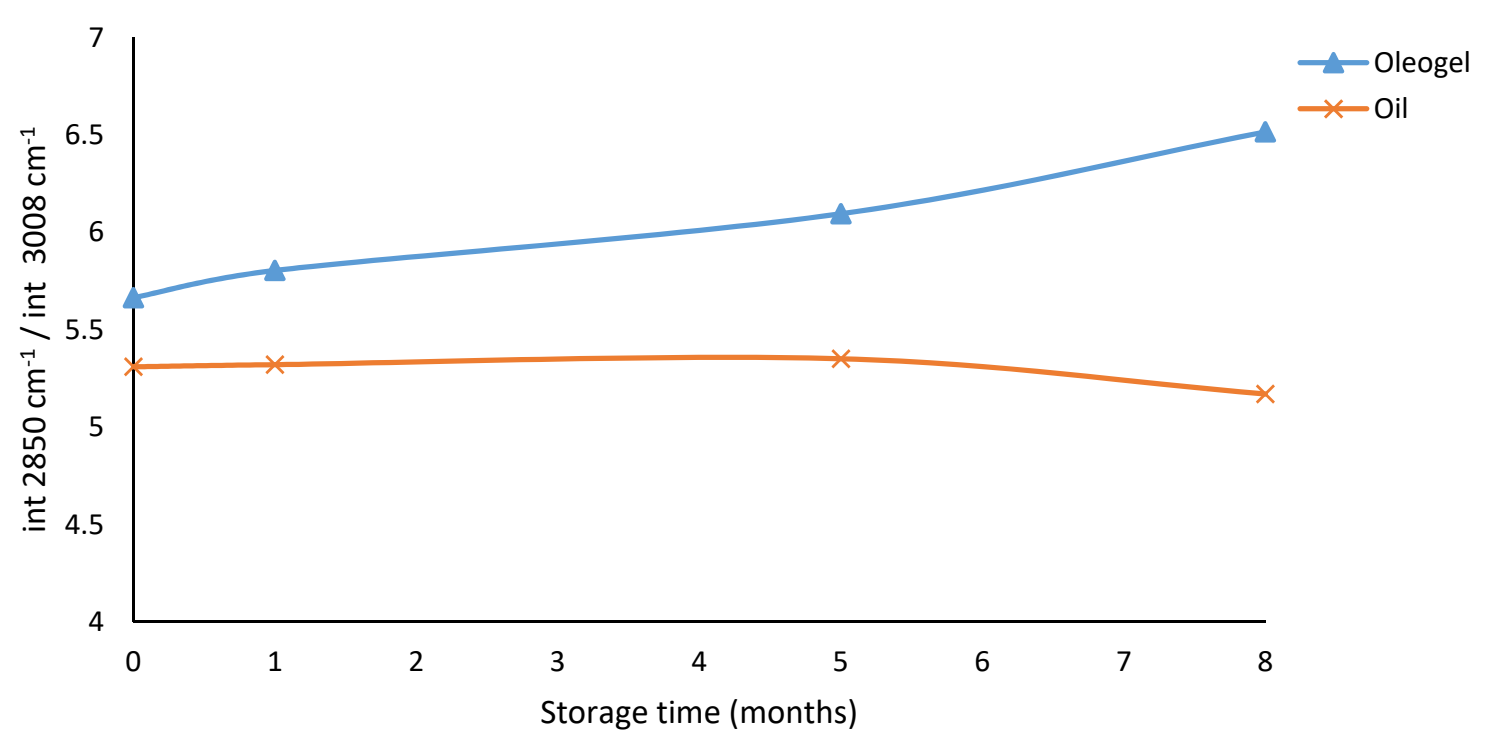

Figure 4. Ratio of intensities $2850 \mathrm{~cm}^{-1} / 3008 \mathrm{~cm}^{-1}$ of the oil and oleogel samples during storage.

\subsection{Principal Component Analysis}

Due to the similarities in terms of chemical composition, the visual comparison of the spectra of pumpkin seed oil and oleogels during storage can lead to inaccurate perceptions and the principal component analysis could better aid in the classification of the samples. PCA was performed in order to group the samples based on their similarities and differences registered in the FTIR analysis. PCA was projected by its principal components (PCs) using raw and SNV-treated FTIR spectral data from 600 to $4000 \mathrm{~cm}^{-1}$. For the raw spectra, PC1 and PC2 accounted, respectively, for $62 \%$ and $25 \%$ of the total data variance. In PCA, the first principle component (PC1) accounts for the most variation, meanwhile the second principle component (PC2) accounts for the samples which fall out of the trend. In the projections of PC1 and PC2, it can be observed that oil samples are well separated from the oleogel samples. As shown in Figure 5a, there is a high similarity between the principal component scores of the raw FTIR spectrum of the oleogel sample in fresh state (ODT1) and those of the oil sample after 1 month of storage (UDT2).

The oil samples of UDT1, UDT2, UDT3 do, however, form a cluster in the upper right side of the plot, while the oil sample stored for 8 months (UDT4) is in the upper left side. A similar trend is also observed for the oleogel samples, leading to the formation another separate cluster. The SNV pre-treated FTIR spectra PC1 horizontal axis explains $60 \%$ of the total variance, while the PC 2 vertical axis explains $20 \%$. The principal component analysis (PCA) scores and loadings, for standard normal variate (SNV) pre-treated FTIR spectra $600-4000 \mathrm{~cm}^{-1}$ also allow the visualization of both oil and oleogel samples stored for 8 months falling out of the trend established by the rest of the sample. The rest of the samples are, however, disseminated closely in the upper and lower right side of the plot, while for the raw FTIR spectra, all the oil samples are in the upper right side and all the oleogel samples are in the lower right side. 


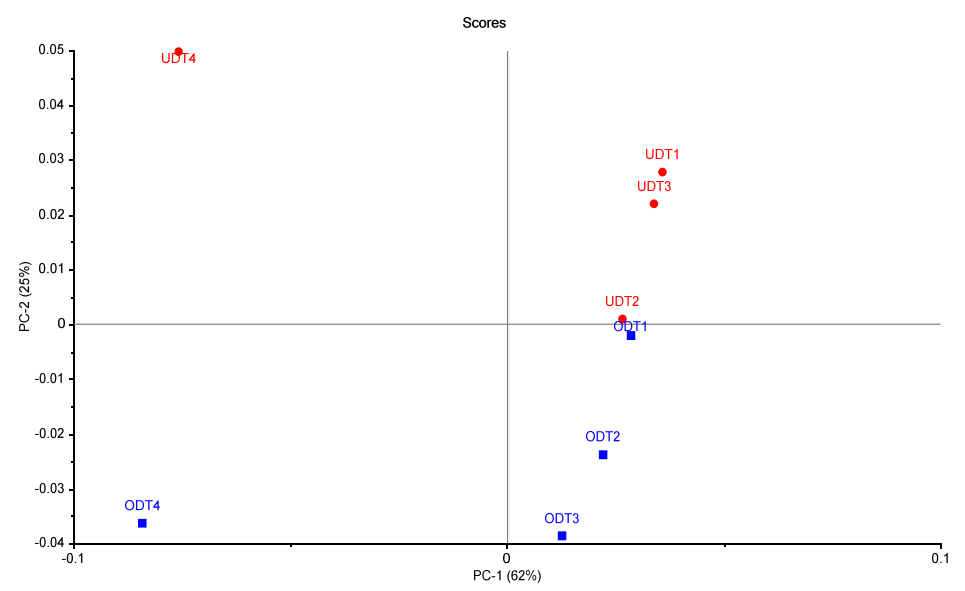

(a)

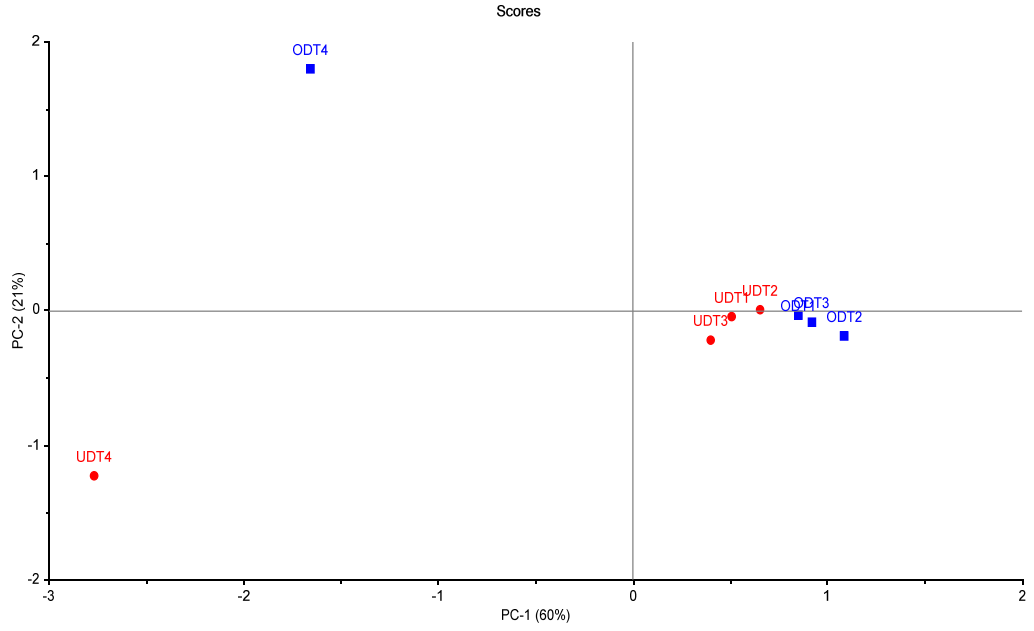

(b)

Figure 5. Principal component analysis (PCA) scores, for raw (a) and SNV-treated (b) FTIR spectra of oils and oleogels in the wave range of $600-4000 \mathrm{~cm}^{-1}$.

\subsection{Partial Least Square Analysis}

A prefeasibility study was conducted with the four samples of the pumpkin seed oil and the four samples of the ternary wax mixture oleogels in different storage moments for predicting the content of the total phenols during the storage of pumpkin oil or oleogels, based on FTIR coupled with partial least square (PLS) calibration and cross-validation models. The leave-one-out cross-validation procedure was performed. The partial least square (PLS) calibration and full cross-validation models for total phenolic prediction by using raw (Figure 6a,b) or SNV (Figure 6c,d) FTIR spectra $600-4000 \mathrm{~cm}^{-1}$ were computed. The PLS calibration and cross-validation models showed encouraging coefficients of determination $\mathrm{R}^{2}$ for raw spectra ( 0.89 for calibration and 0.73 for cross-validation) and SNV-treated spectra ( 0.9 for calibration and 0.86 for cross-validation). The attempt to optimize the spectral region of the PLS model (956-2961 cm $\mathrm{cm}^{-1}$ optimized region based on regression coefficients), revealed $\mathrm{R}^{2}$ increased to 0.93 , indicating a good modelling of the data the calibration model (Figure 7); however, the PLS cross-validation model presented an unsatisfactory coefficient of determination $\mathrm{R}^{2}$ of 0.57 . Consequently, for this prefeasibility study conducted, it was concluded that the best PLS calibration and cross-validation models are obtained when full SNV treated spectra are used (Figure $6 c, d$ ), with a standard error of calibration (SEC) of 0.07 and a standard error of cross-validation (SECV) of 0.1 being obtained. 


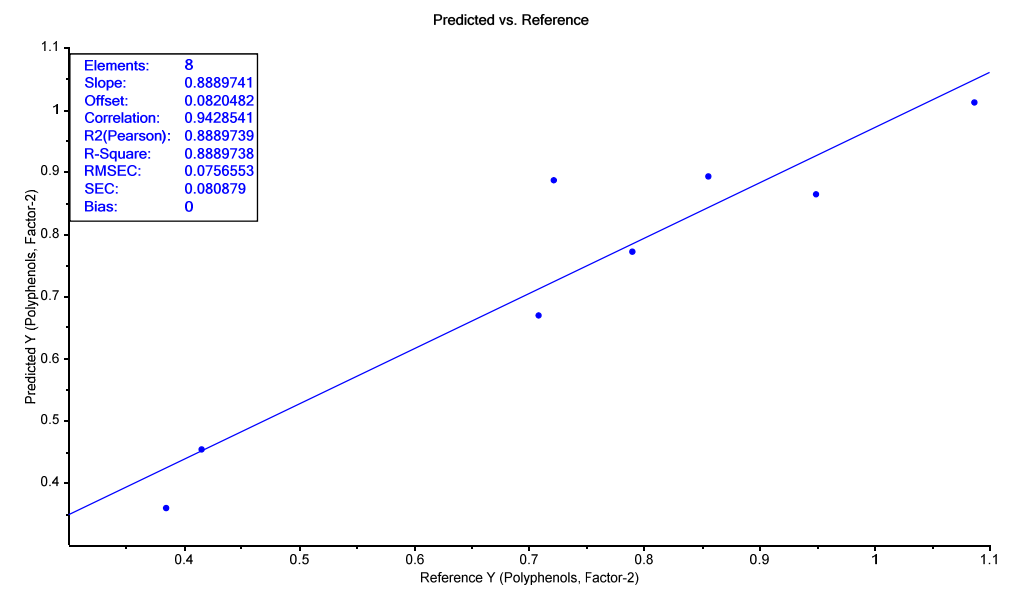

(a)



(c)

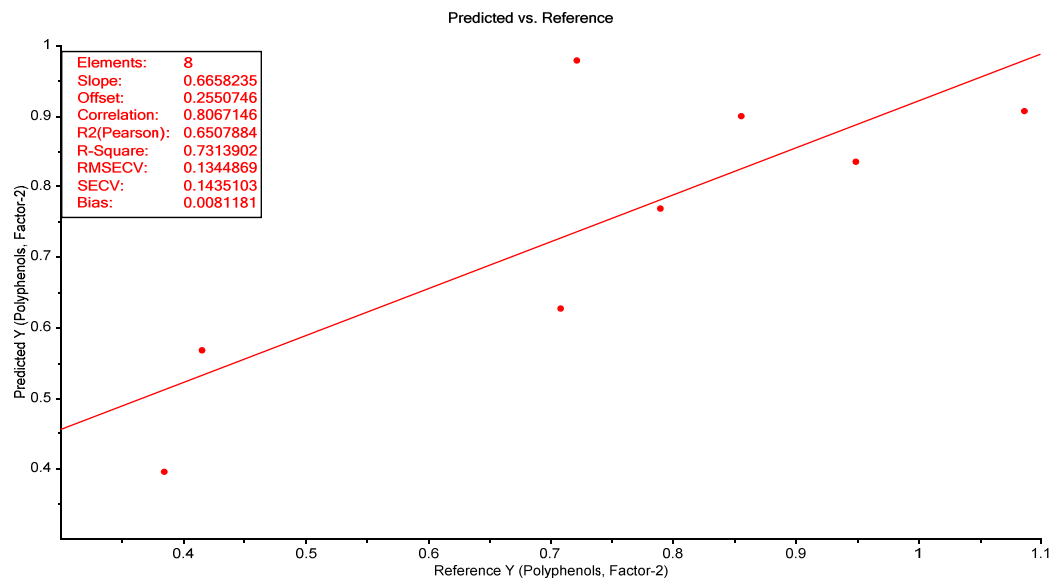

(b)

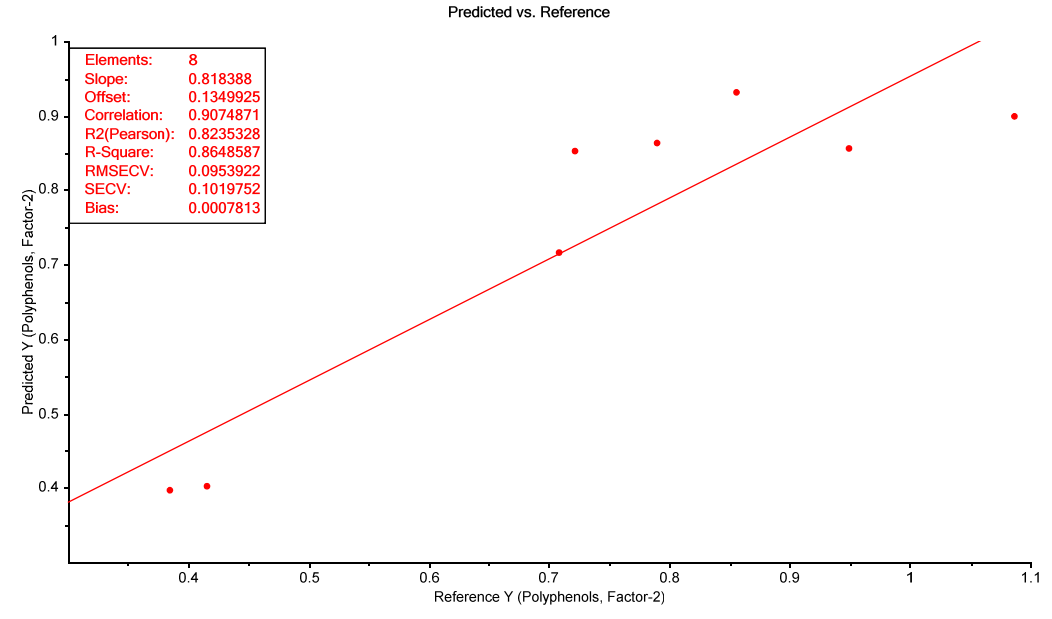

(d)

Figure 6. Partial least square (PLS) calibration $(\mathbf{a}, \mathbf{c})$ and full cross-validation $(\mathbf{b}, \mathbf{d})$ models for total phenolic prediction by using raw $(\mathbf{a}, \mathbf{b})$ or pre-treated standard normal variate $(\mathrm{c}, \mathrm{d})$ FTIR spectra $600-4000 \mathrm{~cm}^{-1}$. 


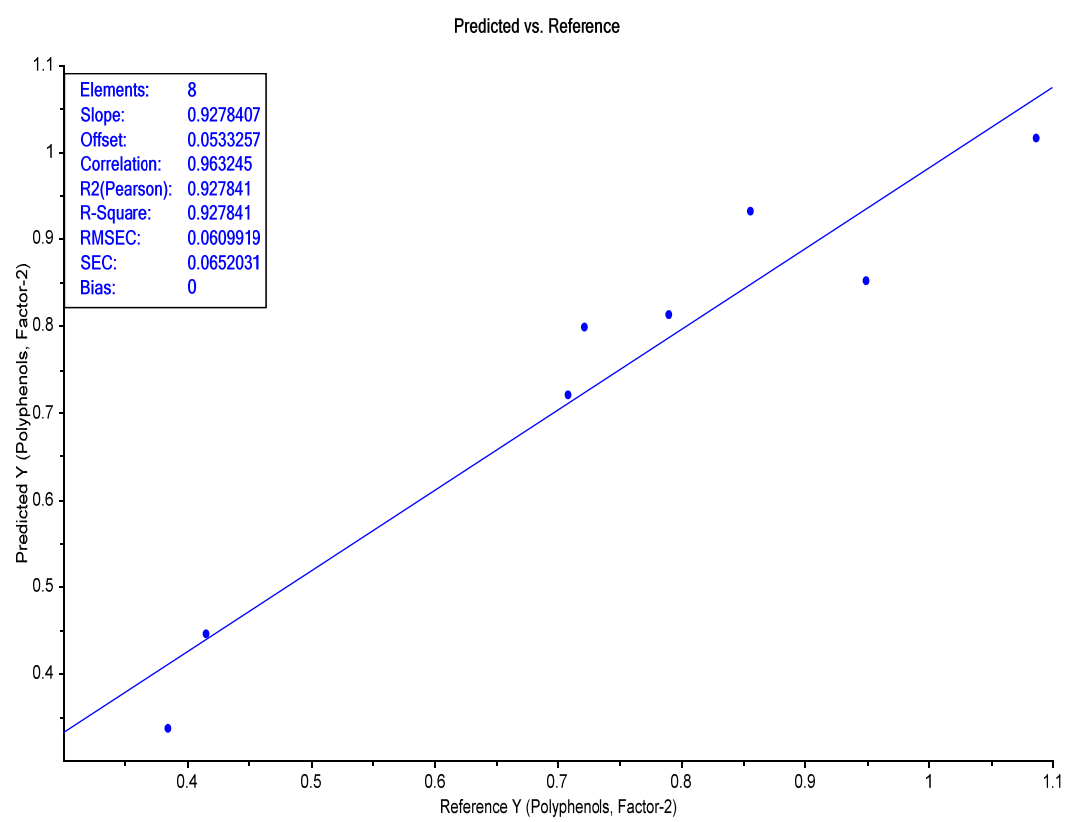

(a)

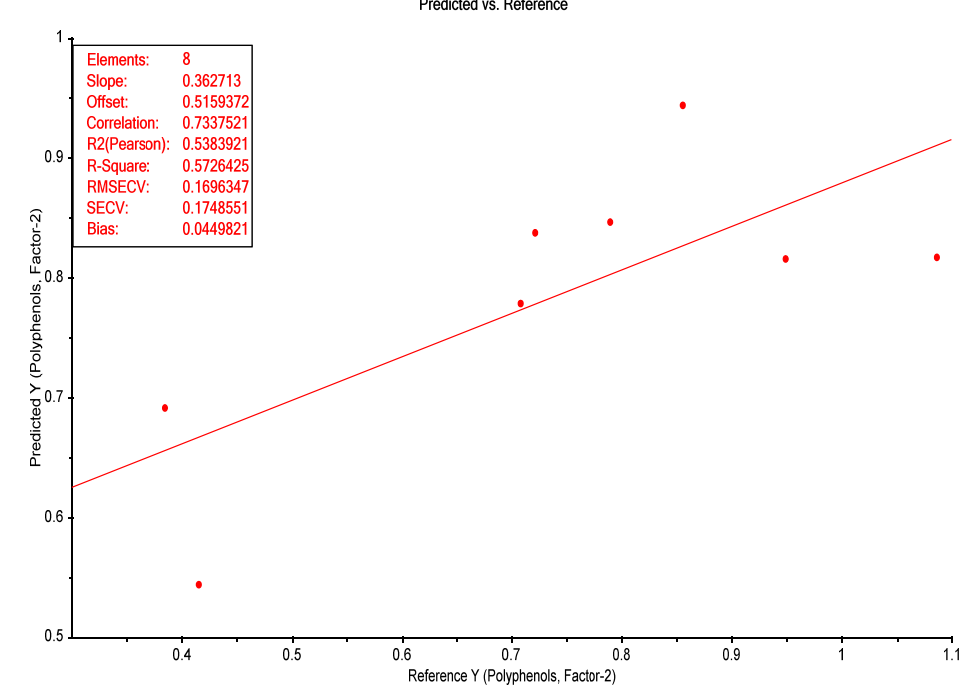

(b)

Figure 7. Partial least square (PLS) calibration (a) and full cross-validation (b) models for total phenolic prediction by using pre-treated standard normal variate (SNV) FTIR spectra-optimum spectral regions selected.

\section{Conclusions}

Liquid-liquid extraction of polyphenols from cold-pressed pumpkin seed oil and oleogel, and their total and individual quantification by HPLC-MS, were performed during ambient storage. Isolariciresinol was the most abundant phenolic compound, but vanillin, caffeic and syringic acids were also identified.

The influence of oleogelation and storage duration on the phenolics dynamics was examined for 8 months. The content of the total polyphenolic compounds was decreased to almost half of the initial content. It is concluded that specific phenolic compounds, such as syringic acid and vanillin, might have been better protected during storage due to oleogelation for 8 months and also isolariciresinol for 5 months. 
FTIR spectroscopy revealed similar trends for the oxidation process in oil and oleogel samples, but after 5 months storage, the oleogel samples became more degraded. The ratio of intensities at $2850 / 3008 \mathrm{~cm}^{-1}$ and the PCA analysis also confirm this. For the raw data, PCA revealed high similarities between the sample of oleogel in fresh state (ODT1) and the oil sample after 1 month of storage (UDT2).

On a pioneered level, due to the low number of samples, the contents of the total phenols during storage of pumpkin oil or oleogels, based on FTIR coupled with partial least square (PLS) calibration and cross-validation models, were studied. It was concluded that the best PLS calibration and cross-validation models were obtained when full SNV-treated spectra were used, with a standard error of calibration (SEC) of 0.07 and a standard error of cross-validation (SECV) of 0.1 being obtained.

Author Contributions: Conceptualization, V.M.; Formal analysis, A.P., A.M., F.R. and F.F.; Funding acquisition, V.M.; Methodology, A.M., F.R. and F.F.; Supervision, S.M., C.S. and V.M.; Writing-original draft, A.P.; Writing-review and editing, V.M. All authors have read and agreed to the published version of the manuscript.

Funding: This work was supported by a grant of Ministry of Research and Innovation, CNCS-UEFISCDI, project number PN-III-P1-1.1-PD-2016-0113, within PNCDI III. The publication fee was supported by funds from the National Research Development Projects to finance excellence (PFE)-37/2018-2020 granted by the Romanian Ministry of Research and Innovation.

Acknowledgments: The authors would like to thank Iasmina Blidar for technical assistance for oleogel sample preparation.

Conflicts of Interest: The authors declare no conflict of interest. The funders had no role in the design of the study; in the collection, analyses, or interpretation of data; in the writing of the manuscript, or in the decision to publish the results.

\section{References}

1. Rezig, L.; Chouaibi, M.; Ojeda-Amador, R.M.; Gomez-Alonso, S.; Salvador, M.D.; Fregapane, G.; Hamdi, S. Cucurbita maxima pumpkin seed oil: From the chemical properties to the different extracting techniques. Not. Bot. Horti Agrobot. Cluj-Napoca 2018, 46, 663-669. [CrossRef]

2. Stevenson, D.G.; Eller, F.J.; Wang, L.; Jane, J.-L.; Wang, T.; Inglett, G.E. Oil and tocopherol content and composition of pumpkin seed oil in 12 cultivars. J. Agric. Food Chem. 2007, 55, 4005-4013. [CrossRef] [PubMed]

3. Parry, J.; Hao, Z.; Luther, M.; Su, L.; Zhou, K.; Yu, L.L. Characterization of cold-pressed onion, parsley, cardamom, mullein, roasted pumpkin, and milk thistle seed oils. J. Am. Oil Chem. Soc. 2006, 83, 847-854. [CrossRef]

4. Andjelkovic, M.; Van Camp, J.; Trawka, A.; Verhé, R. Phenolic compounds and some quality parameters of pumpkin seed oil. Eur. J. Lipid Sci. Technol. 2010, 112, 208-217. [CrossRef]

5. Van Hoed, V.; Sampaio, K.A.; Felkner, B.; Bavec, F.; Scippo, M.L.; Brose, F.; Bavec, M.; Verhé, R. Tocopherols and polyphenols in pumpkin seed oil are moderately affected by industrially relevant roasting conditions. Eur. J. Lipid Sci. Technol. 2017, 119, 1700110. [CrossRef]

6. Yadav, M.; Jain, S.; Tomar, R.; Prasad, G.; Yadav, H. Medicinal and biological potential of pumpkin: An updated review. Nutr. Res. Rev. 2010, 23, 184-190. [CrossRef]

7. Bora, N.S. Beneficial properties of pumpkin seed oil as an antioxidant nutraceutical. EC Pharmacol. Toxicol. 2018, 6, 498-499.

8. Luo, S.-Z.; Hu, X.-F.; Jia, Y.-J.; Pan, L.-H.; Zheng, Z.; Zhao, Y.-Y.; Mu, D.-D.; Zhong, X.-Y.; Jiang, S.-T. Camellia oil-based oleogels structuring with tea polyphenol-palmitate particles and citrus pectin by emulsion-templated method: Preparation, characterization and potential application. Food Hydrocoll. 2019, 95, 76-87. [CrossRef]

9. Jiang, Y.; Liu, L.; Wang, B.; Sui, X.; Zhong, Y.; Zhang, L.; Mao, Z.; Xu, H. Cellulose-rich oleogels prepared with an emulsion-templated approach. Food Hydrocoll. 2018, 77, 460-464. [CrossRef]

10. Co, E.D.; Marangoni, A.G. Chapter 1-Oleogels: An Introduction. In Edible Oleogels, 2nd ed.; Marangoni, A.G., Garti, N., Eds.; AOCS Press: San Diego, CA, USA, 2018; pp. 1-29. [CrossRef] 
11. Samateh, M.; Sagiri, S.S.; Sanni, R.; Chee, C.; Satapathy, S.; John, G. Tuning aesthetic and mechanical properties of oleogels via formulation of enzyme-enabled stereoisomeric molecular gelators. J. Agric. Food Chem. 2019. [CrossRef]

12. Ghosh, M.; Begg, F.; Bhattacharyya, D.K.; Bandyopadhya, N.; Ghosh, M. Nutritional evaluation of oleogel made from micronutrient rich edible oils. J. Oleo Sci. 2017, 66, 217-226. [CrossRef]

13. Nicholson, R.A.; Marangoni, A.G. Enzymatic glycerolysis converts vegetable oils into structural fats with the potential to replace palm oil in food products. Nat. Food 2020, 1-9. [CrossRef]

14. Tavernier, I.; Doan, C.D.; Van de Walle, D.; Danthine, S.; Rimaux, T.; Dewettinck, K. Sequential crystallization of high and low melting waxes to improve oil structuring in wax-based oleogels. RSC Adv. 2017, 7, 12113-12125. [CrossRef]

15. Winkler-Moser, J.K.; Anderson, J.; Felker, F.C.; Hwang, H.S. Physical properties of beeswax, sunflower wax, and candelilla wax mixtures and oleogels. J. Am. Oil Chem. Soc. 2019, 96, 1125-1142. [CrossRef]

16. Hwang, H.S.; Fhaner, M.; Winkler-Moser, J.K.; Liu, S.X. Oxidation of fish oil oleogels formed by natural waxes in comparison with bulk oil. Eur. J. Lipid Sci. Technol. 2018, 120, 1700378. [CrossRef]

17. Wang, X.; Wang, S.-J.; Nan, Y.; Liu, G. The effects of oil type and crystallization temperature on the physical properties of vitamin C-loaded oleogels prepared by an emulsion-templated approach. Food Funct. 2020, 11, 8028-8037. [CrossRef]

18. Martins, A.J.; Cerqueira, M.A.; Cunha, R.L.; Vicente, A. Fortified beeswax oleogels: Effect of $\beta$-carotene on the gel structure and oxidative stability. Food Funct. 2017, 8, 4241-4250. [CrossRef]

19. Masotta, N.E.; Martinefski, M.R.; Lucangioli, S.; Rojas, A.M.; Tripodi, V.P. High-dose coenzyme Q10-loaded oleogels for oral therapeutic supplementation. Int. J. Pharm. 2019, 556, 9-20. [CrossRef]

20. Mao, L.; Wang, D.; Liu, F.; Gao, Y. Emulsion design for the delivery of $\beta$-carotene in complex food systems. Crit. Rev. Food Sci. Nutr. 2016, 58, 770-784. [CrossRef] [PubMed]

21. Hughes, N.E.; Marangoni, A.G.; Wright, A.J.; Rogers, M.A.; Rush, J.W.E. Potential food applications of edible oil organogels. Trends Food Sci. Technol. 2009, 20, 470-480. [CrossRef]

22. Li, L.; Wan, W.; Cheng, W.; Liu, G.; Han, L. Oxidatively stable curcumin-loaded oleogels structured by $\beta$-sitosterol and lecithin: Physical characteristics and release behaviour in vitro. Int. J. Food Sci. Technol. 2019, 54, 2502-2510. [CrossRef]

23. Perez, J.A.V.; Remacho, C.R.; Rodriguez, J.R.; Pulido, J.M.O.; De La Fuente, E.B.; Martinez-Ferez, A. Optimization of Oleogel Formulation for Curcumin Vehiculization and Lipid Oxidation Stability by Multi-response Surface Methodology. Chem. Eng. Trans. 2019, 75, 427-432.

24. Martins, A.J.; Vicente, A.A.; Pastrana, L.M.; Cerqueira, M.A. Oleogels for development of health-promoting food products. Food Sci. Hum. Wellness 2020, 9, 31-39. [CrossRef]

25. Bot, A.; Agterof, W.G.M. Structuring of edible oils by mixtures of $\gamma$-oryzanol with $\beta$-sitosterol or related phytosterols. J. Am. Oil Chem. Soc. 2006, 83, 513-521. [CrossRef]

26. Calligaris, S.; Mirolo, G.; Da Pieve, S.; Arrighetti, G.; Nicoli, M.C. Effect of oil type on formation, structure and thermal properties of $\gamma$-oryzanol and $\beta$-sitosterol-based organogels. Food Biophys. 2013, 9, 69-75. [CrossRef]

27. Rogers, M.A.; Spagnuolo, P.A.; Wang, T.M.; Angka, L. A potential bioactive hard-stock fat replacer comprised of a molecular gel. Food Sci. Nutr. 2017, 5, 579-587. [CrossRef]

28. Li, F. Inulin-Monoglycerides Emulsion Gel as Potential Fat Replacer and Effect of Inulin to Delay Lipid Oxidation. Master's Thesis, Carleton University, Ottawa, ON, Canada, 2019.

29. Freire, M.; Cofrades, S.; Pérez-Jiménez, J.; Gómez-Estaca, J.; Jiménez-Colmenero, F.; Bou, R. Emulsion gels containing n-3 fatty acids and condensed tannins designed as functional fat replacers. Food Res. Int. 2018, 113, 465-473. [CrossRef] [PubMed]

30. Lu, M.; Cao, Y.; Ho, C.-T.; Huang, Q. Development of organogel-derived capsaicin nanoemulsion with improved bioaccessibility and reduced gastric mucosa irritation. J. Agric. Food Chem. 2016, 64, 4735-4741. [CrossRef]

31. Qiu, C.; Huang, Y.; Li, A.; Ma, D.; Wang, Y. Fabrication and characterization of oleogel stabilized by gelatin-polyphenol-polysaccharides nanocomplexes. J. Agric. Food Chem. 2018, 66, 13243-13252. [CrossRef]

32. Rexhepi, F. Antioxidant activity of pumpkin seed oil and its effect on oxidative stability of sunflower oil monitored by FTIR spectroscopy technique. Eur. J. Mater. Sci. Eng. 2020, 5, 51-57. [CrossRef]

33. Pușcas, A.; Mureșan, V.; Socaciu, C.; Muste, S. Oleogels in food: A review of current and potential applications. Foods 2020, 9, 70. [CrossRef] [PubMed] 
34. Martins, A.J.; Vicente, A.A.; Cunha, R.L.; Cerqueira, M.A. Edible oleogels: An opportunity for fat replacement in foods. Food Funct. 2018, 9, 758-773. [CrossRef]

35. Rezig, L.; Chouaibi, M.; Meddeb, W.; Msaada, K.; Hamdi, S. Chemical composition and bioactive compounds of Cucurbitaceae seeds: Potential sources for new trends of plant oils. Process. Saf. Environ. Prot. 2019, 127, 73-81. [CrossRef]

36. Montesano, D.; Blasi, F.; Simonetti, M.S.; Santini, A.; Cossignani, L. Chemical and nutritional characterization of seed oil from Cucurbita maxima L (var. Berrettina) pumpkin. Foods 2018, 7, 30. [CrossRef]

37. Poiana, M.-A.; Alexa, E.; Moigradean, D.; Popa, M. The influence of the storage conditions on the oxidative stability and antioxidant properties of sunflower and pumpkin oil. In Proceedings of the 44th Croatian \& 4th International Symposium of Agriculture, Opatija, Croatia, 16-20 February 2009; pp. 449-453.

38. Ricciutelli, M.; Marconi, S.; Boarelli, M.C.; Caprioli, G.; Sagratini, G.; Ballini, R.; Fiorini, D. Olive oil polyphenols: A quantitative method by high-performance liquid-chromatography-diode-array detection for their determination and the assessment of the related health claim. J. Chromatogr. A 2017, 1481, 53-63. [CrossRef] [PubMed]

39. Gorjanović, S.Ž.; Rabrenović, B.B.; Novaković, M.M.; Dimić, E.B.; Basić, Z.N.; Sužnjević, D.Ž. Cold-pressed pumpkin seed oil antioxidant activity as determined by a DC polarographic assay based on hydrogen peroxide scavenge. J. Am. Oil Chem. Soc. 2011, 88, 1875-1882. [CrossRef]

40. Irnawati, I.; Riyanto, S.; Martono, S.; Rohman, A. The employment of FTIR spectroscopy and chemometrics for authentication of pumpkin seed oil from sesame oil. Food Res. 2019, 4, 42-48. [CrossRef]

41. Rohman, A.; Man, Y.C.; Nurrulhidayah, A. Fourier-transform infrared spectra combined with chemometrics and fatty acid composition for analysis of pumpkin seed oil blended into olive oil. Int. J. Food Prop. 2015, 18, 1086-1096. [CrossRef]

42. Irnawati, I.; Riyanto, S.; Martono, S.; Rohman, A. Determination of sesame oil, rice bran oil and pumpkin seed oil in ternary mixtures using FTIR spectroscopy and multivariate calibrations. J. Food Sci. 2019, 4, 135-142. [CrossRef]

43. Ö̈̆ütcü, M.; Arifoğlu, N.; Yılmaz, E. Preparation and characterization of virgin olive oil-beeswax oleogel emulsion products. J. Am. Oil Chem. Soc. 2015, 92, 459-471. [CrossRef]

44. Yılmaz, E.; Öğütcü, M. Oleogels of virgin olive oil with carnauba wax and monoglyceride as spreadable products. Grasas Y Aceites 2014, 65, e040. [CrossRef]

45. Gómez-Estaca, J.; Herrero, A.M.; Herranz, B.; Álvarez, M.D.; Jiménez-Colmenero, F.; Cofrades, S. Characterization of ethyl cellulose and beeswax oleogels and their suitability as fat replacers in healthier lipid pâtés development. Food Hydrocoll. 2019, 87, 960-969. [CrossRef]

46. Ali, M.A.; Nargis, A.; Othman, N.H.; Noor, A.F.; Sadik, G.; Hossen, J. Oxidation stability and compositional characteristics of oils from microwave roasted pumpkin seeds during thermal oxidation. Int. J. Food Prop. 2017, 20, 2569-2580. [CrossRef]

47. Rohman, A.; Man, Y.C. Application of FTIR spectroscopy for monitoring the stabilities of selected vegetable oils during thermal oxidation. Int. J. Food Prop. 2013, 16, 1594-1603. [CrossRef]

Publisher's Note: MDPI stays neutral with regard to jurisdictional claims in published maps and institutional affiliations.

(C) 2020 by the authors. Licensee MDPI, Basel, Switzerland. This article is an open access article distributed under the terms and conditions of the Creative Commons Attribution (CC BY) license (http://creativecommons.org/licenses/by/4.0/). 